\title{
Mathematical Formulation for Programmers to Select Background and Foreground Colors in Designing Websites
}

\author{
Veeresh Duvvuri, Srinivas Laggoni, Shankar Karinga \\ Guru Nanak Institutions Technical Campus \\ India \\ veeresh.duvvuri@gmail.com \\ laggoni.srinivas589@gmail.com \\ kshankar334@gmail.com
}

\begin{abstract}
For any Website, the look and feel of the page depends on the selection of good color combinations for Background and Foreground Colors, which are represented in RGB format. In most of the cases, the selection of the colors is done by choosing 2 extremely different colors, one being light in color and other dark, for example white and black. The web designers don't know how the color combination looks like unless they are checked or implemented in the system. The proposed methodology can help the designers to choose different combinations of background color and foreground color for a text in a website without executing. The same methodology can be applied for testing the website, with some suggestions or inputs for choosing of the colors. If the color combinations are not appropriate, or they may affect the eye, then the system rejects such color combinations. Because there is no automation testing technique for identification of color for web pages, most of the cases the tester or developer may conflict with each other in selection of the colors.
\end{abstract}

Keywords: Background Color, Foreground Color, Web Testing RGB, Color Combination

Received: 18 December 2017, Revised 30 January 2018, Accepted 8 February 2018

DOI: $10.6025 /$ ijwa/2018/10/2/47-50

(C) 2018 DLINE. All Rights Reserved

\section{Introduction}

A Website is developed using many languages and tools ${ }^{[1]}{ }^{1]}$ such as HTML, CSS, JavaScript, XML, JSP, etc,. But when a markup language is used, the interpreter or any code analyzer cannot identify the properties of the tags included in a program.

For example, consider a tag $<\mathrm{HR}$ Align= "center" Width="75\%" $>$. If a program is used to trace the value of the property "Align" in $<\mathrm{HR}>$ tag, it is not possible.

This problem is applicable to all the tags in HTML. When a Programmer(s) is intended to design a Webpage, the basic consideration will be on the Font and Color related aspects, because they have to include all the functionalities of the project as well as support good User Interface. When testing a project, Application or Website, the functional or structural testing can be

International Journal of Web Applications Volume 10 Number 2 June 2018 
automated keeping all the requirements recorded in $\mathrm{SRS}^{[2]}$. But a testing cannot be automated for some requirements which are not included in the documentation are Font or Color. These concepts will be programmed or tested by the user(s) by manually by selecting different font styles or colors which can be felt good visually.

\section{Literature Survey}

When designing a website, the Programmer will have to choose the language to program, along with the colors need to be considered. It will have affect on the eyes when selecting a range and combination of foreground and background colors.

According to the papers ${ }^{[3][4]}$, the authors examine the effect of web page text/background colour combination on readability, retention, aesthetics, and behavioural intention. Major findings were: (a) Colours with greater contrast ratio generally lead to greater readability; (b) colour combination did not significantly affect retention; (c) preferred colours (i.e., blues and chromatic colours) led to higher ratings of aesthetic quality and intention to purchase; and (d) ratings of aesthetic quality were significantly related to intention to purchase.

In the document ${ }^{[5]}$, study on the design of tablet screen has been proposed, which can be related to the performance of the users. The purpose of this study is to investigate the effects of screen background colors on the brain functions for elderly and young people when they are performing a task on a tablet computer. The results indicate that white color may not be the best choice for a background color of a tablet screen for best performance and attention for both young and elderly people.

Instead of using static markup languages, XML like languages will help in retrieving the background and foreground colors of the websites. Using some of the tools, it is also possible to retrieve the same information ${ }^{[6]}$.

It is a best option to identify the aesthetics of colors selected for the website by the tester or developer. Using the proposed technique, it is formulated that the selection of colors can be made easy before the last phase of software development.

\section{Related Work}

\subsection{Colour Representation Formats}

In HTML, the colour[7] can be specified by using a colour name, an RGB value, or a HEX value.

\section{Colour Names}

Colours of any known Primary Colours or mixed colours for which a name is specified can be used. For example, Blue, Yellow, Orange, Violet, Red can be used. Browsers support 140 standard colour names written in HTML.

\section{RGB Values}

When a color is to be specified as an RGB value, then the rgb (red, green, blue) is used for a combination of colors using the mixture among red, green, and blue colors. Each parameter (red, green, and blue) defines the intensity of the color between 0 and 255.

For example, the function $\mathrm{rgb}(255,0,0)$ is an alternative to represent red color, because red is set to its highest value $(255)$ and the others are set to 0 . Shades of gray are often defined using equal values for all the 3 parameters of the function. For Black, all the parameters of the function must be set to 0 , and for White, all the parameters must be set to 255 .

\section{HEX Values}

Most of the HTML programmers will use HEX value representation of a color, where a color is specified using a hexadecimal value in the form: \#RRGGBB, where RR (red), GG (green) and BB (blue) are hexadecimal values between 00 and FF (same as decimal 0-255).

\section{Methodology}

\subsection{Sample Combinations}

For any document or application or a webpage, the appropriate choice of colours that are to be selected are a Light colour for Background and a Dark colour for the text or Foreground. The choice is universally acceptable, because the light colour spread

48 International Journal of Web Applications Volume 10 Number 2 June 2018


through a large section and will have little strain on the eye, where as the dark colour will be sharp and hence should be of smaller size or portion in the page, so that the content will be immediately recognized by the user easily without much strain on the eye.

Some of the choice of colours that can be preferred for the background and text along with a sample of output is shown in Table 1.

\begin{tabular}{|l|l|l|c|}
\hline S No & Background Color (RRGGBB) & Text Color (RRGGBB) & Sample Output with a text \\
\hline 1 & FFFFF & 000000 & Sample \\
\hline 2 & FFFF00 & FF0000 & Sample \\
\hline 3 & 64 FF64 & 605000 & Sample \\
\hline 4 & FABF8F & $0000 F F$ & Sample \\
\hline 5 & 000000 & FFFFFF & Sample \\
\hline
\end{tabular}

Table 1. Sample Color Combinations for Background and Text

In Table 1, the last entry is the choice against the proposed selection criteria of the colours. But visually it looked good. But if the text or page is viewed for a longer period of time, the eyes will be affected in concentrating and might lead to misinterpretation of the content present.

\subsection{Notations in Formulation}

The $R G B$ colour will be considered in $R R G G B B$ format for both Background as well as Text Colours. Each of the 2 digits are grouped and will be represented with a single character and a subscript denoting whether it belongs to background or text, as $\boldsymbol{R}_{C} \boldsymbol{G}_{C} \boldsymbol{B}_{C}$ for background colour and $\boldsymbol{R}_{T} \boldsymbol{G}_{T} \boldsymbol{B}_{T}$ for text colour. For Signs, $\boldsymbol{R}_{S} \boldsymbol{G}_{S} \boldsymbol{B}_{S}$ can be used.

\subsection{Procedure}

1. Select the foreground and background colours in two variables, $F G$ and $B G$ respectively.

2. Specify the Red, Green, and Blue colours of $F G$ and $B G$; as $R_{T}, G_{T}, B_{T}$ and $R_{C} G_{C} B_{C}$.

3. Subtract corresponding $R, G, B$ colours of $F G$ from $B G$.

4. Calculate the magnitude and signs of the result.

\section{Results and Discussion}

From the sample colours, it is observed with the following analysis.

1. If the signs of all $R_{S}, G_{S}, B_{S}$ are positive and the magnitudes are above 128, then the colours are preferable in making them a pair.

2. If the signs of all $R_{S}, G_{S}, B_{S}$ are negative and the magnitudes are below 128 , then the colours are not preferable in making them a pair.

3. If the signs of any two bits are positive, then the magnitude should not greater than 128 .

4. It is suggestible to have the sign of $R_{S}$ to be positive in all cases.

These results are not based on the selection of colours done genuinely manually, rather they are selected using a mathematical methodology, which is $90 \%$ valid in the output of the selection. The colour pair will not affect much on the eye, even when the users don't approve the colours.

These results also help in designing a web site by the developer, before analysing by the testers. Till now there is no better tool to analyze the colours of a web site than a human eye. But the time taken in analyzing as well as the choice of the colours 
depends on the taste of the developer and tester, which may lead to conflict between them.

\section{Conclusion}

It is suggested that the web designers will know better in choosing the colours for their web sites which will be pleasant for the eyes of the user without much struggle. This methodology helps the designers and testers to choose different combinations of background color and foreground color for a text in a website without executing. In the future enhancement, the choosing of either foreground or background colour results in suggesting different colours for the websites, which will be compatible to the selected color for designing web pages.

\section{References}

[1] Chris Bates, Web Programming: Building Internet Applications, 3, Wiley.

[2] Wikipedia, Software Requirements Specification, Web. https://en.wikipedia.org/wiki/Software_requirements_specification

[3] Richard, H., Hall, Patrick Hanna (2004). The Impact of Web Page Text-Background Colour Combinations on Readability, Retention, Aesthetics and Behavioural Intention, Journal of Behaviour and Information Technology, 3 (3) 183-195.

[4] Richard, H., Hall, Patrick Hanna, (2003). The Effect of Web Page Text-Background Color Combinations on Retention and Perceived Readability, Aesthetics and Behavioral Intention, 9th Americas Conference on Information Systems, p. 277.

[5] Anuardi, Adilin., Shinohara, Hideyuki., Atsuko, K., Yamazaki. (2016). A Pre-study of Background Color Effects on the Working Memory Area of the Brain, Procedia Computer Science, p. 1172-1178.

[6] QTP-How to get font size/color, background color and other attributes of controls, http://motevich.blogspot.in/2007/09/qtpfont-size-color-background-color.html

[7] Web Colors, https://en.wikipedia.org/wiki/Web_colors

\section{Author Biographies}

D. S. S. Veeresh graduated in Computer Science \& Engineering from Swami Ramananda Thirtha Institute of Science \& Technology, Nalgonda, India in 2002, and obtained his M. Tech degree in Software Engineering from Nishitha College of Engineering \& Technology, Hyderabad, India in 2012. He is having the teaching experience of over 12 years. His research interests include Web Services, Wireless Networks, Image Processing.

L. Srinivas received his B.Tech in Computer Science \& Engineering from Swami Ramananda Thirtha Institute of Science \& Technology, Nalgonda, India in 2009, and received his M.Tech degree in Computer Science \& Engineering from MVSR Engineering College, Hyderabad, India in 2012. He is having the teaching experience of 3 years. His research interests include Data Mining, Wireless Networks, Image Processing.

K. Shankar graduated in Computer Science \& Engineering from Jawarharlal Nehru Institute of Technology, Hyderabad, India in 2013, and obtained his M.Tech degree in Computer Science \& Engineering from B.K.B.E.S.G.I, Hyderabad, India in 2015. He is having the teaching experience of 2 years. His research interests include Databases, Network Security. 